\title{
One-dimensional spatially dependent solute transport in semi-infinite porous media: an analytical solution
}

\author{
R. R. Yadav", Lav Kush Kumar \\ Department of Mathematics and Astronomy, Lucknow University, Lucknow-226007, INDIA \\ ${ }^{*}$ Corresponding Author: e-mail: yadav_rr2@yahoo.co.in
}

\begin{abstract}
The present study is an attempt to describe analytical solution of spatially dependent solute transport in one-dimensional semiinfinite homogeneous porous domain. In this mathematical model the dispersion coefficient is considered spatially dependent while seepage velocity is considered exponentially decreasing function of space. Dispersion parameter and velocity are directly proportional to each other. Space dependent retardation factor is also taken. The nature of porous media and solute pollutant are considered chemically non-reactive. Initially porous domain is considered solute free and the input source condition is considered uniformly continuous. A new transformation is introduced to solve the advection dispersion equation. The analytical solution is obtained by using Laplace Transformation Techniques (LTT). The effects of spatial dependence on the solute concentration dispersion of various physical parameters are explained with help of different graphs.
\end{abstract}

Keywords: Diffusion, Seepage velocity, Retardation, Aquifer, Laplace transformation, Pollutant.

DOI: http://dx.doi.org/10.4314/ijest.v9i4.3

\section{Introduction}

A solute transport phenomenon is always an important issue for groundwater contamination which depends on the different chemical and physical processes caused by advection-dispersion. Solute transports in the surfaces and sub surfaces have been one of the most important and interesting research topics in hydrological sciences, engineering and mathematics in the last few decades. Development of an analytical solutions for groundwater pollution problems are major interesting area for civil engineers, hydrologist, chemical engineer and mathematicians, because these solutions are much helpful to understand or explain the mechanism of contaminant transport, measure the field parameters related to solute transport, predict the movement of contaminant plumes, and verify the numerical results. Most of the theories of solute transports in the porous media included limited assumptions are available in published literature. Groundwater velocities and hydrodynamic dispersion coefficients are key parameters for description of solute transport in porous media. Solute transport in soil, reservoir, and aquifer is generally governed by advection-dispersion equation. This equation infer from two laws one is Darcy's law and other is conservation of mass (Freeze and Cherry, 1979).

A number of mathematical models describing groundwater flow and solute transport in homogeneous and heterogeneous porous domain have been developed in the past and are available in literature. Huang et al. (1996) obtained a general analytical solution for solute transport in one-dimension with asymptotic scale-dependent dispersion in heterogeneous porous domain with assumption that the dispersivity increases linearly with space. Logan (1996) obtained analytical solutions of advection dispersion equation for solute transport in heterogeneous porous media. Leij et al. (1993) obtained an analytical solution assuming velocity and dispersion coefficient constant with space and time in three-dimensional porous domain. Fry et al. (1993) developed analytical solution of one-dimensional advection-dispersion equation in homogeneous isotropic porous medium. Van Kooten (1995) developed a method for predicting the advective-dispersive transport of a contaminant toward a well in a confined aquifer. Yule and Gardener (1978) obtained analytical solution to describe solute transport for line source neglecting longitudinal dispersion. 
Perez Guerrero et al. (2009) presents an analytical solution of dispersion equation with constant coefficients in both transient and steady state conditions by using integral transform and change of variable techniques in a finite spatial domain. Hunt (2006) has given solution for instantaneous, continuous and steady pollution sources in uniform groundwater and Zoppou and Knight (1997) obtained an analytical solutions for the spatially dependent dispersion Yates (1990, 1992) obtained analytical solutions of advection dispersion equation in one-dimension considering dispersion coefficient as linear and exponential increasing. Zhang $e t$ al. (1994) presents an analytical solution of dispersion equation with distance-dependent dispersion coefficient. Pang and Hunt (2001) obtained analytical solutions for advection dispersion equation with scale-dependent dispersion where as Leij et al. (1991) presents several analytical solutions of advection-dispersion equation for three-dimensional in semi-infinite homogeneous porous media with unidirectional solute flow assuming uniform initial concentration with first and third type boundary conditions. Aral and Liao (1996) developed analytical solutions for the two-dimensional advection dispersion equation while Hunt (1998) discussed one, two and three-dimensional advection dispersion equation with scale-dependent dispersion coefficients. Su et al. (2005) developed closed form solutions of dispersion equation with time and scale-dependent disparity for solute transport in heterogeneous porous media while Singh and Kumari (2014) obtained one-dimensional solute transport for prediction of contaminant along unsteady groundwater flow.

Huang et al. (2006) presents an evidence of scale-dependent fractional advection-dispersion in one-dimension. Yadav et al. (2010) present analytical solutions of advection dispersion equation in semi-infinite longitudinal porous domain with temporally dependent coefficients. A linear advection-diffusion equation with variable coefficients in a one-dimensional semi-infinite medium is solved analytically using a Laplace transformation technique, for two dispersion problems: temporally dependent dispersion along a uniform flow and spatially dependent dispersion along a non-uniform flow by Jaiswal et al. (2009). Longitudinal solute transport from a pulse type source along temporally and spatially dependent flow was discussed by Yadav et at. (2012). A power series solution for solute transport in a convergent flow field with scale-dependent advection-dispersion equation with variable coefficients was obtained by Chen et al. (2003). Yadav et al. (2010) present solution of one dimensional temporally dependent advection dispersion equation in homogeneous porous media. Jaiswal and kumar (2011) obtained analytical solutions of advectiondispersion equation in one-dimension with variable coefficients for two cases one time dependent and other spatially dependent dispersion while analytical solutions of one-dimensional advection-diffusion equation with temporally dependent coefficients was obtained by Jaiswal et al. (2011). Sanskrityayn et al. (2016) obtained analytical solution of advection dispersion equation with spatially and temporally dependent dispersion using Green's function. Gao et al. (2010) was presented a new mobile-immobile model to account for solute transport with scale-dependent dispersion in heterogeneous porous media and the dispersivity was assumed to be a function of travel distance, also the linear adsorption and the first-order degradation of solute were considered. The linear and exponential functions were chosen to describe the relationship between dispersivity and distance. Kumar and Yadav (2015) developed analytical solution for conservative solute transport in one dimensional heterogeneous porous medium and solute dispersion parameter is considered uniform, while the seepage velocity spatially dependent. Singh and Das (2015) present an analytical solution of one-dimensional scale dependent solute dispersion in semi-infinite heterogeneous porous medium. Sharma et al. (2016) presented the behavior of solute transport through mobile-immobile soil column based on the laboratory study. Singh $e t$ al. (2012) present solution of one-dimensional solute transport with space-time-dependent contaminant concentration along uniform flow in a semi-infinite homogeneous porous medium. Singh and Chatterjee (2016) presented analytical solution for nonpoint source of concentration in semi-infinite aquifer using Laplace transform technique.

In the present study, an analytical solution of solute transport equation is solved using Laplace transform technique in a semiinfinite, homogeneous porous media. The hydrodynamic dispersion, seepage velocity and retardation factor are considered exponentially deceasing function of space dependent. Retardation factor and dispersion parameter are directly proportional to the velocity. The space dependent retardation factor indicates that entire mass of the dissolved substance is not uniform. Continuous input point source is introduced at the beginning of the domain. Initially porous domain is considered solute free; it means there is no concentration present in the medium. Concentration gradient is considered zero at infinity. Solution is demonstrated graphically with a hypothetical input data taken from the previous published works. Ground water velocity ranges from $2 \mathrm{~m} / \mathrm{year}$ to $2 \mathrm{~m} / \mathrm{day}$, intermediate values are taken in present study (Todd, 1980).

\section{Mathematical formulation of the Problem}

The advection-dispersion equation in one-dimension may be written as follows (Freeze and Cherry, 1979 and Bear, 1972),

$$
\mathrm{R}(\mathrm{x}, \mathrm{t}) \frac{\partial \mathrm{C}}{\partial \mathrm{t}}=\frac{\partial}{\partial \mathrm{x}}\left(\mathrm{D}(\mathrm{x}, \mathrm{t}) \frac{\partial \mathrm{C}}{\partial \mathrm{x}}-\mathrm{u}(\mathrm{x}, \mathrm{t}) \mathrm{C}\right)
$$

In which $\mathrm{C}\left[\mathrm{ML}^{-3}\right]$ is the solute concentration. $\mathrm{D}\left[\mathrm{L}^{2} \mathrm{~T}^{-1}\right]$ and $\mathrm{u}\left[\mathrm{LT}^{-1}\right]$ are the dispersion coefficient and seepage velocity respectively. These may be constants or functions of time or space or both. The left hand side of equation (1) is represent change in solute concentration with time and $\mathrm{R}$ is retardation factor which is a dimensionless quantity. The right-hand side of the equation (1) represents the influence of the dispersion on the solute concentration distribution by first term and the change of the solute concentration due to advective solute transport by second term. Velocity at the scale of pores causes a solute particle to spread 
from initial position. This spreading phenomenon is described at the Darcy scale through dispersion coefficient. In view of established concept hydraulic conductivity causes water velocity at each point in the porous media.

\subsection{Uniform Input Point Source Condition}

The model simulates concentration along one-dimensional space dependent flow through homogeneous semi-infinite porous domain. Groundwater flow is considered along the $x$-axis, means the direction of the flow of water is from $\mathrm{x}=0$ to $\mathrm{x} \rightarrow \infty$. Initially the porous domain is supposed to be solute free this means that before solute injection in the domain there is no concentration present in the domain. A continuous mass injection of solute is introduced into the aquifer at $\mathrm{x}=0$. To formulate the present problem mathematically the initial and boundary conditions are given by Eqs.(2), (3) and (4).

$$
\begin{array}{ccc}
\mathrm{C}(\mathrm{x}, \mathrm{t})=0 & ; \quad \mathrm{t}=0, \mathrm{x} \geq 0 \\
\mathrm{C}(\mathrm{x}, \mathrm{t})=\mathrm{C}_{0} & ; \quad \mathrm{t}>0, \mathrm{x}=0 \\
\frac{\partial \mathrm{C}(\mathrm{x}, \mathrm{t})}{\partial \mathrm{x}}=0 & ; \quad \mathrm{t} \geq 0, \mathrm{x} \rightarrow \infty
\end{array}
$$

Eq.(2) represent initially there is no concentration in the domain. The boundary condition (3) represents the continuous injection of pollutant while boundary condition (4) represents solute concentration gradient is zero at the end of the domain.

\subsection{Analytical Solution}

Let us write

$$
\left.\begin{array}{l}
u(x, t)=u_{0} e^{-m x} \\
D(x, t) \propto u \quad \Rightarrow \\
R(x, t) \propto u \quad \Rightarrow \quad R(x, t)=D_{0} e^{-m x} \\
R(x, t)=R_{0} e^{-m x}
\end{array}\right\}
$$

where $\mathrm{u}_{0}, \mathrm{D}_{0}$ and $\mathrm{R}_{0}$ are initial constants of velocity, dispersion and retardation factor respectively. $\mathrm{m}\left[\mathrm{L}^{-1}\right]$ is the flow resistance coefficient whose dimension is inverse of space. Thus parameter $\mathrm{mx}$ is the dimensionless variable. $\mathrm{m}=0$ represents the spatially independent parameters.

Substituting these values in Eq. (1) we have

$$
\mathrm{R}_{0} \mathrm{e}^{-\mathrm{mx}} \frac{\partial \mathrm{C}}{\partial \mathrm{t}}=\frac{\partial}{\partial \mathrm{x}}\left(\mathrm{D}_{0} \mathrm{e}^{-\mathrm{mx}} \frac{\partial \mathrm{C}}{\partial \mathrm{x}}-\mathrm{u}_{0} \mathrm{e}^{-\mathrm{mx}} \mathrm{C}\right)
$$

This reduces into following equation

$$
\mathrm{R}_{0} \frac{\partial \mathrm{C}}{\partial \mathrm{t}}=\mathrm{D}_{0} \frac{\partial^{2} \mathrm{C}}{\partial \mathrm{x}^{2}}-\mathrm{U}_{0} \frac{\partial \mathrm{C}}{\partial \mathrm{x}}+\gamma_{0} \mathrm{C}
$$

where $\mathrm{U}_{0}=\left(\mathrm{u}_{0}+\mathrm{mD}_{0}\right)$ and $\gamma_{0}=\mathrm{mu}_{0}$

Now Laplace transformation technique is used to get the analytical solution. For which to eliminate the convection term from Eq.(6), we introduce a new dependent variable $\mathrm{K}(\mathrm{x}, \mathrm{t})$ by following transformation.

$$
\mathrm{C}(\mathrm{x}, \mathrm{t})=\mathrm{K}(\mathrm{x}, \mathrm{t}) \exp \left\{\frac{\mathrm{U}_{0}}{2 \mathrm{D}_{0}} \mathrm{x}-\frac{1}{\mathrm{R}_{0}}\left(\frac{\mathrm{U}_{0}^{2}}{4 \mathrm{D}_{0}}-\gamma_{0}\right) \mathrm{t}\right\}
$$

With help of above transformation Eq.(7), the boundary value problem Eqs. $(6,2,3)$ and (4) reduces into following three equations in terms of $\mathrm{K}(\mathrm{x}, \mathrm{t})$ as follows

$$
\begin{array}{cc} 
& \mathrm{R}_{0} \frac{\partial \mathrm{K}}{\partial \mathrm{t}}=\mathrm{D}_{0} \frac{\partial^{2} \mathrm{~K}}{\partial \mathrm{x}^{2}} \\
\mathrm{~K}(\mathrm{x}, \mathrm{t})=0 & ; \mathrm{t}=0, \mathrm{x} \geq 0 \\
\mathrm{~K}(\mathrm{x}, \mathrm{t})=\mathrm{C}_{0} \exp \left(\delta^{2} \mathrm{t}\right) & ; \mathrm{t}>0, \mathrm{x}=0 \\
\frac{\partial \mathrm{K}(\mathrm{x}, \mathrm{t})}{\partial \mathrm{x}}+\frac{\mathrm{U}_{0}}{2 \mathrm{D}_{0}} \mathrm{~K}=0 & ; \mathrm{t} \geq 0, \mathrm{x} \rightarrow \infty
\end{array}
$$

where $\delta^{2}=\frac{1}{\mathrm{R}_{0}}\left(\frac{\mathrm{U}_{0}^{2}}{4 \mathrm{D}_{0}}-\gamma_{0}\right)$

Applying Laplace transformation on it which comprise of following three equations 


$$
\begin{array}{lr}
\frac{\mathrm{d}^{2} \overline{\mathrm{K}}}{\mathrm{dx}^{2}}-\frac{\mathrm{pR}_{0}}{\mathrm{D}_{0}} \overline{\mathrm{K}}=0 \\
\overline{\mathrm{K}}(\mathrm{x}, \mathrm{p})=\frac{\mathrm{C}_{0}}{\mathrm{p}-\delta^{2}} \\
\frac{\mathrm{d} \overline{\mathrm{K}}}{\mathrm{dx}}+\frac{\mathrm{U}}{2 \mathrm{D}_{0}} \overline{\mathrm{K}}=0 & ; \mathrm{x} \rightarrow \infty
\end{array}
$$

where $\overline{\mathrm{K}}=\int_{0}^{\infty} \mathrm{K}(\mathrm{x}, \mathrm{t}) \mathrm{e}^{-\mathrm{pt}} \mathrm{dt}$ and $\mathrm{p}$ is Laplace parameter.

The general solution of Eq.(12) is written as

$$
\overline{\mathrm{K}}(\mathrm{x}, \mathrm{p})=\mathrm{c}_{1} \exp \left(-\mathrm{x} \sqrt{\frac{\mathrm{pR} \mathrm{R}_{0}}{\mathrm{D}_{0}}}\right)+\mathrm{c}_{2} \exp \left(\mathrm{x} \sqrt{\frac{\mathrm{pR} \mathrm{R}_{0}}{\mathrm{D}_{0}}}\right)
$$

Using boundary conditions Eqs.(13) and (14) we have the particular solution as

$$
\overline{\mathrm{K}}(\mathrm{x}, \mathrm{p})=\frac{\mathrm{C}_{0}}{\mathrm{p}-\delta^{2}} \exp \left(-\mathrm{x} \sqrt{\frac{\mathrm{pR} \mathrm{R}_{0}}{\mathrm{D}_{0}}}\right)
$$

Applying inverse Laplace transformation on it, using appropriate tables (Van Genuchten and Alves, 1982) and the necessary transformation defined as above backwards, we get

$$
\begin{aligned}
C(x, t) & =\frac{C_{0}}{2} \exp \left(\frac{x U_{0}-x \sqrt{U_{0}^{2}-4 \gamma_{0} D_{0}}}{2 D_{0}}\right) \times \operatorname{erfc}\left(\frac{R_{0} x-t \sqrt{U_{0}^{2}-4 \gamma_{0} D_{0}}}{2 \sqrt{D_{0} R_{0} t}}\right) \\
& +\frac{C_{0}}{2} \exp \left(\frac{x U_{0}+x \sqrt{U_{0}^{2}-4 \gamma_{0} D_{0}}}{2 D_{0}}\right) \times \operatorname{erfc}\left(\frac{R_{0} x+t \sqrt{U_{0}^{2}-4 \gamma_{0} D_{0}}}{2 \sqrt{D_{0} R_{0} t}}\right)
\end{aligned}
$$

where $\mathrm{U}_{0}=\left(\mathrm{u}_{0}+\mathrm{mD}_{0}\right)$ and $\gamma_{0}=\mathrm{mu}_{0}$.

This is the desired solution of one-dimensional advection-dispersion equation in semi-infinite homogeneous porous medium with spatially dependent coefficients.

\section{Result and Discussions}

The analytical solution obtained as in Eq. (17) is demonstrated with set of input data to understand the solute concentration distribution behavior in the domain. The chosen set of data is taken from the experimental and theoretical published literatures (Todd, 1980, Jaiswal et.al 2009). The domain is considered semi-infinite but the solute concentration decreases with position and time in a finite domain at different values of time. The solute concentration distribution values $\mathrm{C} / \mathrm{C}_{0}$ are calculating by taking reference concentration as $\mathrm{C}_{0}=1.0$, in a finite longitudinal domain $0 \leq \mathrm{x}(\mathrm{km}) \leq 5.0$ at various parameters. The various parameters are taken to illustrate the concentration profile and are given in concern figures. Figure 1 is drawn for the parameters $\mathrm{D}_{0}=1.05 \mathrm{~km}^{2} / \mathrm{yr}, \mathrm{R}_{0}=1.15, \mathrm{~m}=0.001(\mathrm{~km})^{-1}$ at different time. It demonstrates that the trends of contaminant concentration profiles are similar and decreases with position. At particular position the concentration level is lower for lower time and higher for larger time. It shows that tendency of solute concentration is reducing in nature with time and distance travelled in presence of source contaminant. It may help to rehabilitate the solute concentration. Figure 2 is drawn for various retardation factor for the parameters $\mathrm{D}_{0}=1.05 \mathrm{~km}^{2} / \mathrm{yr}, \mathrm{t}=1.0 \mathrm{yr}, \mathrm{m}=0.001(\mathrm{~km})^{-1}$. It depict that the solute concentration profile at particular position is lower for larger retardation value while higher for smaller retardation value. Figure 3 is drawn for various flow resistance for the parameters $\mathrm{D}_{0}=1.05 \mathrm{~km}^{2} / \mathrm{yr}, \mathrm{t}=1.0 \mathrm{yr}, \mathrm{R}_{0}=1.15$. It demonstrates that the contaminant concentration profile at particular position is faster for the higher flow resistance and slower for lower flow resistance. The tendency of solute concentration is reducing in nature with distance travelled in presence of source contaminant. Figure 4 is drawn for various dispersion parameter for the parameters $\mathrm{t}=1.0 \mathrm{yr}, \mathrm{R}_{0}=1.15, \mathrm{~m}=0.001(\mathrm{~km})^{-1}$. It represents that the contaminant concentration profile at particular position is lower for the lower dispersion parameter and higher for higher dispersion parameter with distance travelled in presence of source contaminant. From graphs we conclude that the contaminant concentration reduces with increasing retardation factor and 
increasing with increasing dispersion parameter. The tendency of contaminant concentration with distance travelled in presence of source contaminant and time is reducing in nature. It may help to predict the harmless concentration level in the domain.

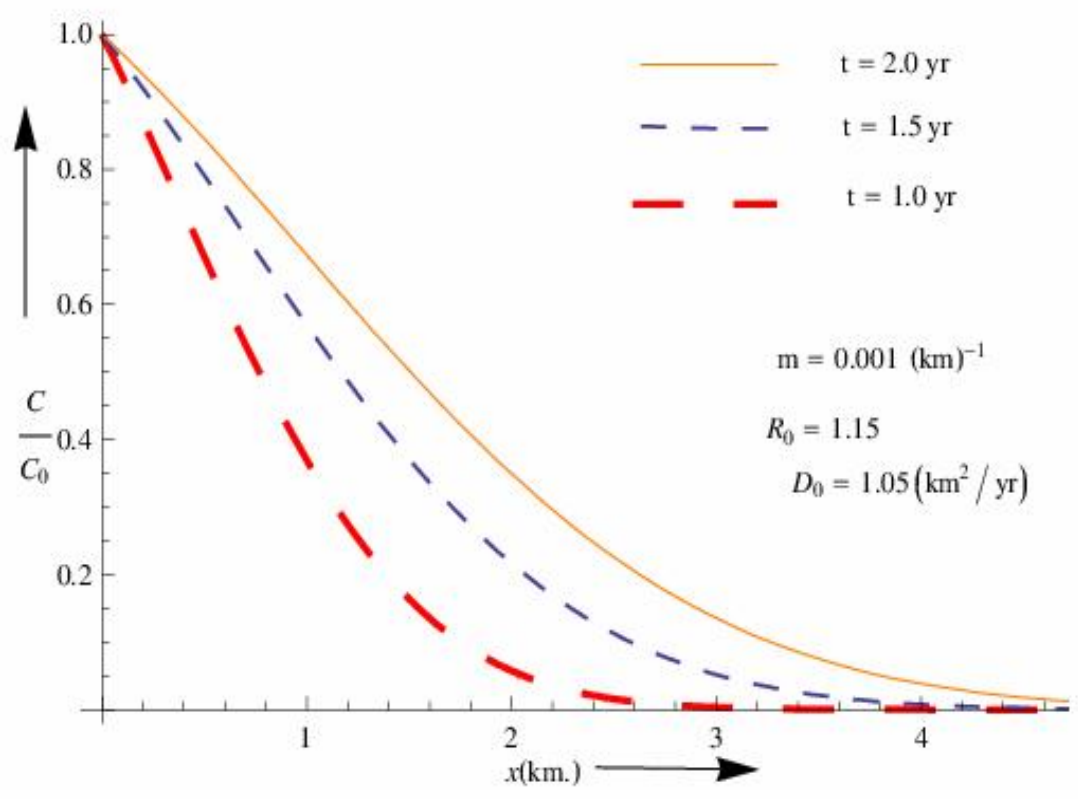

Figure 1. Dimensionless concentration profiles at time $\mathrm{t}(\mathrm{yr})=1.0,1.5,2.0$.

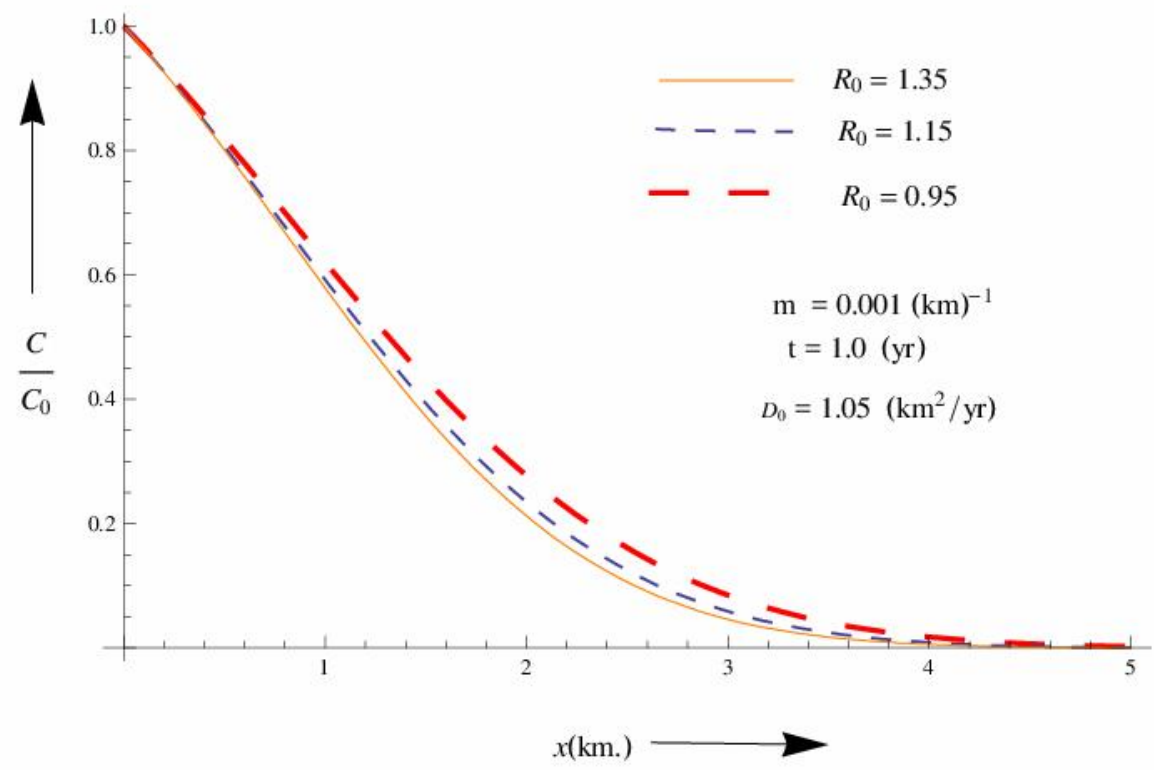

Figure 2. Dimensionless concentration profiles at $\mathrm{R}_{0}=0.95,1.15,1.35$ for time $\mathrm{t}(\mathrm{yr})=1.0$. 


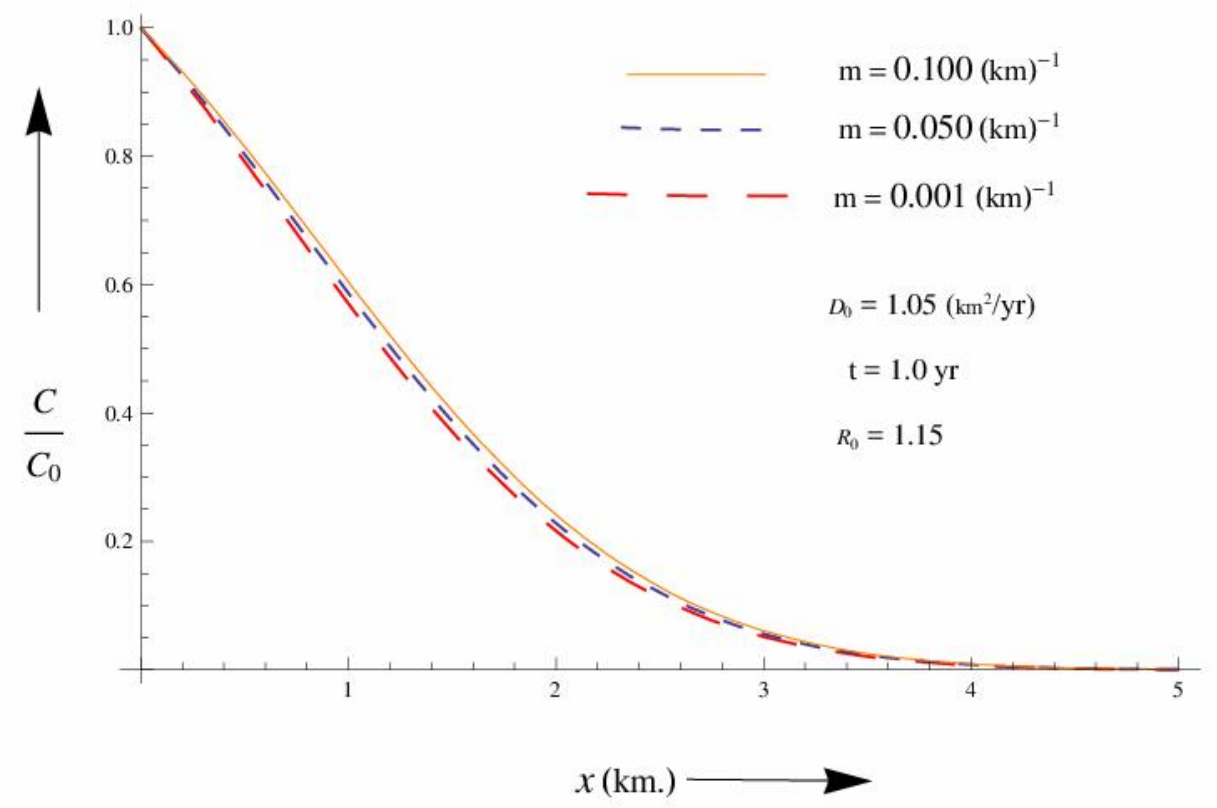

Figure 3. Dimensionless concentration profiles at $\mathrm{m}(\mathrm{km})^{-1}=0.001,0.050,0.100$ for time $\mathrm{t}(\mathrm{yr})=1.0$

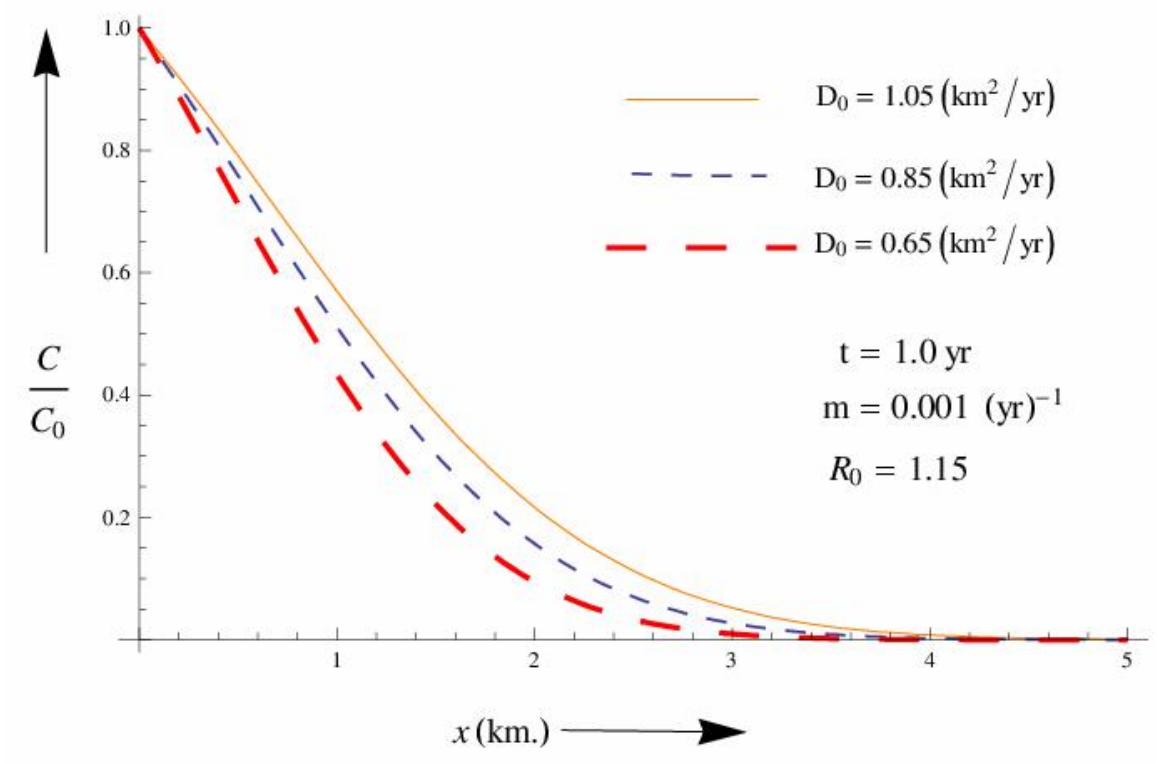

Figure 4. Dimensionless concentration profiles at $\mathrm{D}_{0}\left(\mathrm{~km}^{2} / \mathrm{yr}\right)=0.65,0.85,1.05$ for time $\mathrm{t}(\mathrm{yr})=1.0$

\section{Conclusions}

A mathematical model is presented for accounting concentration distribution in semi-infinite homogenous porous media using Laplace transform technique. In this work, advective dispersive term, transient velocity and retardation factor are considered spatially dependent function. Dispersion parameter and retardation factor are considered directly proportional to the seepage velocity while seepage velocity is assumed exponentially deceasing function of space. The solution in all possible combinations of spatially dependent coefficients is compared with help of graphs. The trends of solute concentration with distance travelled in presence of source contaminant and time are reducing in nature which may help to understand rehabilitation tendency of the contaminated aquifer in the domain which may help as the primary predictive tools in groundwater management system. From the 
obtained solution of dispersion equation and graphs, we also conclude that the contaminant concentration reduces with increasing retardation factor where as increases with increasing time, dispersion parameter and flow resistance coefficients and vice-versa. The tendency of concentration profile is reducing in nature with distance travelled in presence of source contaminant and time. It may help to predict the harmless concentration level in the domain. The obtained solution of advection dispersion equation can be imposed to predict the field problems where hydrological properties of the medium, initial and boundary conditions are same as or can be approximated by ones considered in this study. Results obtained in the proposed problem demonstrate the several features of scale dependent dispersion and retardation in homogeneous porous media

\section{Acknowledgement}

This research did not receive any specific grant from funding agencies in the public, commercial, or not-for profit sectors.

\section{References}

Aral, M.M., and Liao, B., 1996. Analytical solutions for two-dimensional transport equation with time-dependent dispersion coefficients, Journal of Hydrologic Engineering, Vol.1, No.1, pp.20-32.

Bear, J., 1972. Dynamics of Fluid in Porous Media, Elsvier Publ. Co., New York.

Chen, J.S., Liu, C.W., Hsu, H. T. and Liao, C.M., 2003. A Laplace transform power series solution for solute transport in a convergent flow field with scale-dependent dispersion, Water Resources Research, Vol.39, No.8, pp.1229-1238.

Freeze, R. A., and Cherry, J. A., 1979. Groundwater, Prentice-Hall, Englewood Cliffs, NJ.

Fry, V. A., Istok, J. D., and Guenther, R.B., 1993. An analytical solution to the solute transport equation with rate-limited desorption and decay, Water Resources Research, Vol.29, No.9, pp.3201-3208.

Gao, G., Zhan, H., Feng, S., Fu, B., Ying Ma, Y., and Huang, G., 2010. A new mobile-immobile model for reactive solute transport with scale dependent dispersion, Water Resources Research, Vol.46, No.8, W08533(1-16).

Guerrero, J. S. P., Pimentel, L. C. G., Skaggs, T. H., and van Genuchten, M. Th., 2009. Analytical solution of the advectiondiffusion transport equation using a change of variable and integral transform technique, International Journal of Heat and Mass Transfer, Vol.52, pp.3297-3304.

Huang, G., Huang, Q., and Zhan, H., 2006. Evidence of one-dimensional scale-dependent fractional advection-dispersion, Journal of Contaminant Hydrology, Vol.85, pp.53-71.

Huang, K., Van Genuchten, M. T., and Zhang, R., 1996. Exact solutions for one-dimensional transport with asymptotic scaledependent dispersion, Applied Mathematical Modelling, Vol.20, No.4, pp.298-308.

Hunt, B., 1998. Contaminant source solutions with scale-dependent dispersivity, Journal of Hydrologic Engineering, Vol.3, No.4, pp.268-275.

Hunt, B., 2006. Asymptotic solutions for one-dimensional dispersion in rivers, Journal of Hydraulic Engineering, Vol.132, pp. 8793.

Jaiswal, D. K., Kumar, A., Kumar, A., and Yadav, R.R., 2009. Analytical solutions for temporally and spatially dependent solute dispersion of pulse type input concentration in one-dimensional semi-infinite media, Journal of Hydro-environment Research, Vol. 2, No.4, pp.254-263.

Jaiswal, D.K. and Kumar, A., 2011. Analytical solutions of advection-dispersion equation for varying pulse type input point source in one-dimension, International Journal of Engineering Science and Technology, Vol.3, No.1, pp.22-29.

Jaiswal, D.K., Kumar, A. and Yadav, R.R., 2011. Analytical solution to the one- dimensional advection-diffusion equation with temporally dependent coefficients, Journal of Water Resource and Protection, Vol.3 No.1, pp.76-84.

Kumar, A. and Yadav, R.R., 2015. One dimensional solute transport for uniform and varying pulse type input point source through heterogeneous medium, Environmental Technology, Vol.36, No.4, pp.487-495.

Leij, F. J., Skaggs, T. H., and Van Genuchten, M. Th., 1991. Analytical solution for solute transport in three-dimensional semiinfinite porous media, Water Resources Research, Vol.27, No.10, pp.2719-2733.

Leij, F. J., Toride, N., and Van Genuchten, M.Th., 1993. Analytical solutions for non equilibrium solute transport in threedimensional porous media, Journal of Hydrology, Vol.151, pp.193-228.

Logan, J. D., 1996. Solute transport in porous media with scale dependent dispersion and periodic boundary conditions, Journal of Hydrology, Vol.184, No.3-4, pp.261-276.

Pang, L., and Hunt, B., 2001. Solutions and verification of a scale-dependent dispersion model, Journal of Contaminant Hydrology, Vol. 53, pp.21-39.

Sanskrityayn, A., Bharati, V. K., and Kumar, N., 2016. Analytical solution of ADE with spatiotemporal dependence of dispersion coefficient and velocity using green's function method, Journal of Groundwater Research, Vol.5, No.1, pp.24-31.

Sharma, P. K., Shukla, S. K., Choudhary, R. and Swami, D., 2016. Modeling for solute transport in mobile-immobile soil column experiment, ISH Journal of Hydraulic Engineering, Vol. 22, No.2, pp.204-211.

Singh, M. K., and Chatterjee, A., 2016. Solute dispersion in a semi-infinite aquifer with specified concentration along an arbitrary plane source, Journal of Hydrology, Vol.541, pp. 928-934. 
Singh, M. K., and Kumari, P., 2014. Analytical solution of contaminant transport in two-dimensional homogeneous semi-infinite aquifer, National Conference on Sustainable Development of Groundwater Resources in Industrial Regions.

Singh, M.K. and Das, P., 2015. Scale dependent solute dispersion with linear isotherm in heterogeneous medium, Journal of Hydrology, Vol. 520, pp.289-299.

Singh, M.K., Ahamad, S., and Singh, V.P., 2012. Analytical solution for one-dimensional solute dispersion with time-dependent source concentration along uniform groundwater flow in a homogeneous porous formation, Journal of Engineering Mechanics, Vol.138, No.8, pp.1045-1056.

Su, N., Sander, G.C., Liu, F., Anh, V., and Barry, D.A., 2005. Similarity solutions for solute transport in fractal porous media using a time and scale-dependent dispersivity, Applied Mathematical Modeling, Vol.29, pp.852-870.

Todd, D. K., 1980. Groundwater Hydrology, $2^{\text {nd }}$ Ed. John Wiley, New York.

Van Genuchten, M. Th., and Alves, W. J., 1982. Analytical solutions of the one-dimensional convective-dispersive solute transport equation, Technical Bulletin No. 1661, U.S. Department of Agriculture, Washington, DC.

Van Kooten, Jaco J. A., 1995. An asymptotic method for a pumping well, Advances in Water Resources, Vol.18, No.5, pp. 295313.

Yadav, R. R., Jaiswal, D. K., Yadav, H. K., and Gulrana, 2010. One-dimensional temporally dependent advection-dispersion equation in porous media: Analytical solution, Natural resource modelling, Vol.23, No.4, pp.521-539.

Yadav, R.R, Jaiswal, D.K, Gulrana and Yadav, H.K, 2010. Analytical solution of one dimensional temporally dependent advection dispersion equation in homogeneous porous media, International Journal of Engineering, Science and Technology, Vol.2, No.5, pp.141-148.

Yadav, S. K., Kumar, K., and Kumar, N., 2012. Horizontal solute transport from a pulse type source along temporally and spatially dependent flow: Analytical solution, Journal of Hydrology, Vol. (412-413), pp.193-199.

Yates, S. R., 1990. An analytical solution for one-dimensional transport in heterogeneous porous media, Water Resources Research, Vol.26, No.10, pp.2331-2338.

Yates, S. R., 1992. An analytical solution for one-dimensional transport in porous media with an experimental dispersion function, Water Resources Research, Vol.28, No.8, pp.2149-2154.

Yule, D. F., and Gardner, W. R., 1978. Longitudinal and transverse dispersion coefficients in unsaturated Plainfield sand, Water Resources Research, Vol.14, pp.582-588.

Zhang, R., Huang, K., and Xiang, J., 1994. Solute movement through homogeneous and heterogeneous soil columns, Advances in Water Resources, Vol.17, No.5, pp.317-324.

Zoppou, C., and Knight, J.H., 1997. Analytical solutions for advection and advection diffusion equation with spatially variable coefficients, Journal of Hydraulic Engineering, Vol.123, pp.144-148.

\section{Biographical notes}

Dr. R. R. Yadav is a Professor in Department of Mathematics \& Astronomy, Lucknow University, Lucknow, India. He has more than twenty five years research/teaching experience at undergraduate and postgraduate level. He has published more than three dozen research papers of international and national journals.

Lav Kush Kumar is doing Ph. D. under supervision of Dr. R.R. Yadav in the Department of Mathematics \& Astronomy, Lucknow University, Lucknow, India.

Received March 2016

Accepted June 2017

Final acceptance in revised form June 2017 